\title{
Is pneumatic balloon dilation safe and effective primary modality of treatment for post-sleeve gastrectomy strictures? A retrospective study
}

Aneesh Shrihari Dhorepatil, Daniel Cottam*, Amit Surve, Walter Medlin, Hinali Zaveri, Christina Richards and Austin Cottam

\begin{abstract}
Background: The optimal treatment of sleeve strictures has not been agreed upon at the current time. At our institution, we began using pneumatic balloon dilation to help resolve these obstructions in 2010. Herein we report our experience with pneumatic balloon dilation for the treatment of sleeve strictures.

Methods: From Jan 2010 to Dec 2016 we retrospectively reviewed our prospectively kept database for patients who developed a Laparoscopic Sleeve Gastrectomy (LSG) stricture within 90 days of surgery. If the stricture was found, then we dilated all our patients initially at $30 \mathrm{~mm}$ at $10 \mathrm{PSI}$ for 10-20 min (14.5 min average) and increased the balloon size (30-40 mm) and duration (10-30 min) in subsequent sessions if the first session was unsuccessful.

Results: The review found that 1756 patients underwent either LSG or the first step of a Laparoscopic Duodenal Switch (LDS) (1409 LSG \& 356 LDS). Of the 1756 patient 33 patients (24 underwent LSG, and 9 underwent LDS) developed a stricture as a complication of LSG. The average age of the patients was $46.4( \pm 9.6)$ years, and the average BMI was 43.7 ( \pm 6.4 ). The most common location for stricture was mid-body of the sleeve (54.5\%). The average time from the primary surgery to diagnosis and first pneumatic dilation was 5.6 months $( \pm 6.8)$ and 5.9 months ( \pm 6.6) respectively. We successfully used pneumatic dilation in 31 (93.9\%) of these patients to relieve the stricture.
\end{abstract}

Conclusion: We conclude that pneumatic dilation is an effective procedure in patients with post sleeve gastrectomy stricture.

Keywords: Laparoscopic sleeve gastrectomy, Loop-duodenal switch, Strictures, Pneumatic balloon dilation, Endoscopic management, Bariatric

\section{Background}

Prevalence of laparoscopic sleeve gastrectomy (LSG) for morbid obesity increased from 0 to $37 \%$ of the total world interventions for weight loss surgery between 2003 and 2013 [1]. This increase in popularity is attributed to its lower complication rates and safety as a procedure [2].

Despite the LSG's rapidly rising popularity, strictures have remained an ongoing problem with an occurrence rate of $0.1-3.9 \%$ [1]. Strictures are usually divided into

* Correspondence: drdanielcottam@yahoo.com

Bariatric Medicine Institute, 1046 East 100 South, Salt Lake City, UT 84102, USA

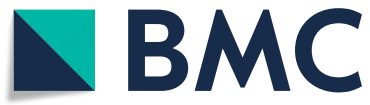

(c) The Author(s). 2018 Open Access This article is distributed under the terms of the Creative Commons Attribution 4.0 International License (http://creativecommons.org/licenses/by/4.0/), which permits unrestricted use, distribution, and reproduction in any medium, provided you give appropriate credit to the original author(s) and the source, provide a link to the Creative Commons license, and indicate if changes were made. The Creative Commons Public Domain Dedication waiver (http://creativecommons.org/publicdomain/zero/1.0/) applies to the data made available in this article, unless otherwise stated. early strictures and late strictures. Patients with early strictures present within the first few weeks following the surgery complaining of dysphagia, vomiting, food intolerance, rapid weight loss, and regurgitation of either food or saliva. These are often pseudo strictures caused either as a result of post-operative edema or hematoma formation.

Late strictures, which occur $>1$ month from the time of surgery are usually true strictures [3]. They are usually caused by ischemia, retraction due to scarring, or misalignment during stapling [3].

Treatment of LSG strictures is controversial. When to intervene, when to dilate, with what to dilate, and when 
to place metallic stents are all active areas of debate [4]. When these non-invasive methods fail, when to perform surgery and which type of surgery to perform, whether it's a simple seromyotomy or strictureplasty or more invasive approaches to some bypass procedure, all lack consensus [5, 6].

In this article, we share one of the largest case series of LSG strictures treated with pneumatic balloon dilation as a primary modality of treatment. We also present a sustainable management plan using pneumatic balloon dilation as the primary modality of treatment for LSG strictures.

\section{Methods}

This study has been approved by Quorum Review- Independent review board (QR\# 31353), prior to data collection. 1409 cases of LSG and 356 cases of LDS were retrospectively reviewed from a prospectively collected database from Jan 2010 to Dec 2016. Demographic data for all patients was collected. This included pre-operative BMI, age, weight, and co-morbidities.

Thirty-three patients $(1.8 \%)$ that presented with dysphagia, nausea, vomiting, or food intolerance after LSG or LDS with documented stricture on endoscopy or upper gastrointestinal (UGI) contrast study were considered eligible for pneumatic dilation. An area of narrowing, slow passage, or frank obstruction on an UGI were defined as a stricture. Similarly, a stricture was defined as a small, unsurpassable stretch of the lumen when using a $9.8 \mathrm{~mm}$ endoscope with or without symptoms, especially if the lumen is $6 \mathrm{~mm}$ or less [3]. We included all patients who demonstrated a stricture of gastric origin and underwent at least $20 \mathrm{~mm}$ of dilation into the study. We excluded all patients with strictures of non-gastric origin and patients undergoing a revision surgery of gastric bypass.

We used endoscopic pneumatic balloon dilation as a primary modality of treatment and repeated the procedure if symptoms did not resolve. Patients were informed of a surgical alternative in cases requiring repeated dilations.

\section{Technique of pneumatic dilation}

We performed all our endoscopies under general anesthesia. Initially, an endoscope is passed down to the level of the stricture under direct vision. The stricture's position is then confirmed on fluoroscopy and marked with a simple paper clip on the anterior abdominal wall. After confirming the stricture, the scope is then passed distally to the stricture and into the distal small bowel. A MAXXWIRE (MeritMedica Endotek) metallic guide-wire is passed through the scope and using fluoroscopic guidance placed in the distal small bowel, and the EGD scope is removed keeping the guide-wire in place. Then using the guidewire and fluoroscopic guidance we slide the Rigiflex II Balloon (Boston Scientific, Marlborough, Massachusetts) into position using the previously placed paper clip as a reference.

The balloon is then inflated under fluoroscopic and endoscopic visualization to $30 \mathrm{~mm}$ for an average of 14.5 min per patient. Optical view through the balloon and re-inspection endoscopy was used to confirm the resolution of the stricture. Depending on clinical improvement and the patients' decision, repeat dilations were performed up to a maximum of $40 \mathrm{~mm}$, with a minimum interval of 2 weeks between 2 dilations.

\section{Technique for LSG and LDS [7]}

All surgeries were done by the three surgeons at the Bariatric Medicine Institute at a single hospital in Salt Lake City with identical technique. Out technique for both the LSG and LDS has been described previously [7].

Briefly, the LSG is created by stapling alongside a 40 French bougie. No patient in the study had his or her staple line oversewn or staple line reinforced. The staple line in all patients is started approximately $5 \mathrm{~cm}$ from the pylorus and ended at the angle of His. Each patient has a visual inspection of the hiatus to evaluate for a hiatal hernia with the simultaneous repair if a defect is found.

The loop duodenal switch procedure begins with an identical technique as a LSG. Following this, the gastroepiploic vessels are divided from the end of the sleeve staple line past the pylorus to the point where the perforating vessels from the pancreas enter the duodenum. This is 2 to $4 \mathrm{~cm}$ beyond the pylorus. The duodenum is divided with an Endo GIA stapler (Medtronic). Then the ileocecal valve is identified, and $300 \mathrm{~cm}$ of small bowel are measured and marked for point of anastomosis. The small bowel is then connected to the proximal duodenum.

\section{Results}

Out of 1756 patients who underwent sleeve gastrectomy or LDS, 33 patients were included in this study. Twenty-seven patients were female, and 6 patients were male. The average age of the patients was $46.4( \pm 9.6)$ years, and the average BMI was $43.7( \pm 6.4)$.

Sixty-five percent of the patients followed up at 1 year and the excess body weight loss (EBWL\%) at 1 year was $66.1 \%(62.6-69.7 \%)$.

24 patients underwent laparoscopic sleeve gastrectomy, and 9 patients underwent a LDS. Of 33 patients, 15 patients $(45.4 \%), 5$ patients (15.1\%), and 9 patients (27.2\%) had diabetes mellitus (DM), positive smoking history, and hiatal hernia repair, respectively. After endoscopy and/or Upper GI series, in $54.5 \%(18 / 33)$ 
patients the stricture was localized to the Mid-Body, $30.2 \%(10 / 33)$ were noted to have it at the incisura, and $15.2 \%(5 / 33)$ had it in the upper $1 / 3$ of the sleeve. The average time from surgery to diagnosis was 5.6 months \pm 6.8 months. The average duration of primary surgery to the first pneumatic dilation was 5.9 months $(+/-6.6)$. The average duration from primary surgery to the second dilation was 8.6 months $(+/-7.1)$. Overall only 2 patients from the study group were dilated 3 times. The average duration of primary surgery to the third dilation was 19 months $(+/-19.7)$. The average balloon size used for the first dilation was $32.1 \mathrm{~mm}(+/-6.8)$; for the second dilation it was $35 \mathrm{~mm}(+/-5.2)$; and for the third dilation it was $40 \mathrm{~mm}$.

The mean duration of dilation was $14.5 \mathrm{~min}(+/-$ $7.5 \mathrm{~min}$ ). In the 21 patients who required a single dilation for symptom resolution, the mean duration was $15.6 \mathrm{~min}(+/-6.6)$. Of the 8 patients that required a second dilation for symptom resolution, the mean duration of dilation in the first dilation was $14.6 \mathrm{~min}(+/-8.3)$ and in their second dilation was $20 \mathrm{~min}(+/-8 \mathrm{~min})$. The 2 patients who required 3 dilations for symptom resolution were dilated for $10 \mathrm{~min}$ during the first dilation; $22.5 \mathrm{~min} \mathrm{(+/-}$ $10.6 \mathrm{~min})$ during their second dilation, and $30 \mathrm{~min}$ during their third dilation. 93.9\% (31/33) patients had complete resolution of symptoms after pneumatic balloon dilation. $67.7 \%(21 / 31)$ required only 1 dilation, $25.8 \%(8 / 31)$ required 2 dilations, and $6.4 \%(2 / 31)$ required 3 dilations. Of the remaining $6.1 \%(2 / 33)$ patients, 1 patient required surgical intervention for symptom resolution, and 1 patient required a fcSEMS stent.

The location of the stricture, operative details, and the outcomes following pneumatic dilation can be seen in Table 1.

\section{Discussion}

Strictures following a LSG is a very rare complication with a reported incidence of $0.1-3.9 \%$ [1]. Due to this rarity, there are few studies which thoroughly explore its management. Strictures usually present about 6 weeks after surgery [8], and are characterized by persistent dysphagia, nausea, vomiting, and food regurgitation following surgery. Early identification and management lead to better outcomes [8]. Our study is one of the largest case series detailing the magnitude of the complication and explores the efficacy of pneumatic balloon in its management.

According to many authors, the most common site of stricture is at the incisura [5, 9-11], but we observed that the most common location of the stricture was at the Mid-Body of the narrow sleeve (54.5\%) followed by the incisura (30.2\%) and then the upper 1/3rd of the sleeve (15.2\%). This correlates with similar studies conducted by Donatelli et al. [12] and Rebibo et al. [13]. The question as to why we have more strictures in the mid-body just like Donatelli and Rebibo we believe relates to technique, specifically, the way the assistant pulls on the stomach while stapling. However, since the rate of stricture formation was only $2 \%$, it will be several years and almost a thousand patients before we can definitively say whether or not our change in technique has made a difference.

Excess body weight loss achieved at 1 year after LSG can range from 50 to $70 \%[7,14,15]$. We believe with early recognition and treatment, the presence of a stricture does not alter the pattern and degree of excess body weight loss achieved by LSG. This can be seen in the EBWL\% achieved by the patients in this study, which was $66.1 \%$.

Graded Pneumatic Balloon Dilation has been introduced as an effective and safer alternative to surgery for strictures [16, 17]. The primary aim of pneumatic balloon dilation is to pull apart the fibrosed muscular fibers. The pneumatic balloon, due to its rigid structure, achieves the high radial force of expansion [10]. Our study's use of higher initial balloon size and increased duration time ensures the initial few minutes of dilation help tear the muscular fibers while the longer duration overcomes the elasticity of the fibrosis which has invariably occurred at the site of the stricture.

The magnitude of balloon dilation required for most effective management has not yet been defined. Most authors recommend $30 \mathrm{~mm}$ as the diameter of the balloon during the initial dilation for effective management of the stricture [3, 11]. Shnell et al. [10] demonstrated a relative lack of clinical success using the $20 \mathrm{~mm}$ balloon, and Donatelli et al. [12] and Rebibo et al. [13] showed higher success rates using the $30 \mathrm{~mm}$ balloon. In this study, we too used the $30 \mathrm{~mm}$ balloon during the first dilation. We believe dilating with up to $40 \mathrm{~mm}$ balloon is safe and effective even during the initial dilation. We dilated 8 patients with $40 \mathrm{~mm}$ balloons during their initial dilation and reported no adverse events associated with the pneumatic dilation. As far as efficacy is concerned, 50\% (4/8) reported resolution of symptoms after a single dilation and $50 \%(4 / 8)$ required repeated dilation. In our experience, the $30 \mathrm{~mm}$ balloon is the safer and wiser choice as an initial dilator. We believe there isn't a significant increase in efficacy by using a larger balloon as an initial dilator. The decision to use a larger balloon must be patient specific and should be taken after visualizing the stricture intra-operatively. Further studies comparing the $30 \mathrm{~mm}$ balloon and the $40 \mathrm{~mm}$ are required to determine the benefit of one over the other.

Compared to other studies, which document up to $1 \mathrm{~min}$ as the duration of dilation, this study has 


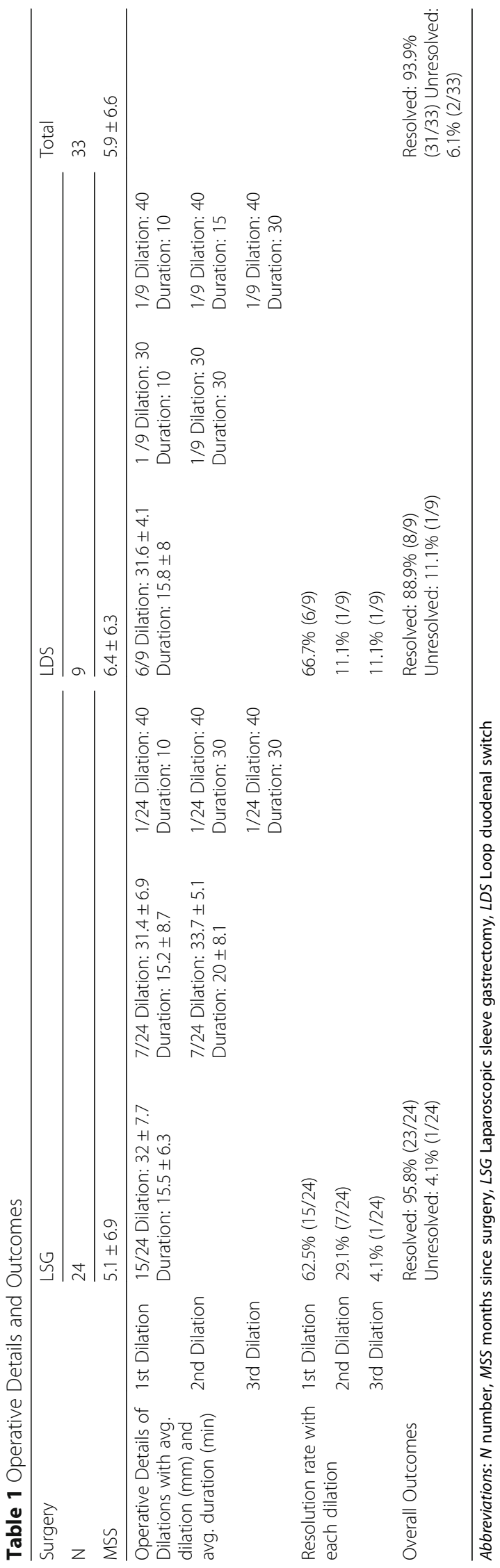


demonstrated a mean duration of $14.5 \mathrm{~min}(+/-7.5 \mathrm{~min})$. The increased duration of dilation leads to increased long-term patency of the previously strictured sleeve. We propose it leads to the lesser need for repeat dilations and lesser need for operative intervention to treat the stricture. We achieved successful resolution of symptoms in 93.9\% of our patients only using pneumatic dilation. $67.7 \%$ of the patients with reported resolution of symptoms required just a single dilation. This could be the reason for our higher success rate as compared to other studies in the literature $[9-12,18]$. Moreover, the patients who achieved symptom resolution after a single dilation were subjected to a longer duration of dilation (15.3 $\mathrm{min}$ ) as compared to those who required a second dilation $(14.6 \mathrm{~min})$ \{during their first dilation\}. The longer balloon dilation times are not unheard of as Zundel dilated his sleeve strictures for a mean of $20 \mathrm{~min}$ [3]. In reality, the basic science and retrospective data are lacking in this regard, and there are no papers comparing dilation time and the incidence of complication. Our paper clearly showed that longer dilation times lead to better outcomes. However, we would never object to the argument that the appropriate dilation time and size are still open for vigorous debate.

Recently, Deslauriers et al. successfully performed dilation in $56 \%$ of his patients with sleeve stenosis [19]. Of these, $73 \%$ only needed single dilation, while $44 \%$ of the patients who were unsuccessful needed conversion to gastric bypass. All the successful cases needed a maximum of 3 interventions. Our success rate was higher and failure rate was lower than the one reported in this study. However, the success rate with single dilation was very similar between the two studies (73\% vs $68 \%)$. Similarly, Nath A et al. [20] performed hydrostatic balloon dilation in 33 patients with gastric sleeve narrowing. He used 10-18 $\mathrm{mm}$ of balloon dilation for $1 \mathrm{~min}$. Resolution was seen in $69 \%$ of his patients; $39.4 \%$ after first dilation, $15.2 \%$ after 2 nd dilation and $15.2 \%$ after third dilation. Two patients $(6 \%)$ had no improvement at all. His resolution rate was also lower compared to ours; however, it is important to note that size of his dilation was much smaller compared to the other published studies [10, 12, 13, 21].

Complex strictures sometimes require a different approach. The treatment options include fcSEMS stent and revision surgeries including pyloroplasty, stricturoplasty [22], or revision bariatric surgery [6]. fcSEMS are currently used for short periods to fine-tune narrowed sleeves [4]. Stents are most useful in refractory strictures, which have failed multiple attempts at dilation [4]. Unfortunately, stent migration is a very real complication and is reported in a very high number of cases [4]. This leads to repositioning in a majority of cases and operative stent removal in others.
In this study, we had 1 patient who required a stent. The patient underwent stent placement because of diverticulum formed due to gastric dilation, which required a partial gastrectomy. The patient experienced persistent pain and dysphagia 1 week after stent placement. After endoscopy, it was revealed that the stent had migrated into the esophagus, which had caused an intussusception of the esophagus and had led to an obstruction. We proceeded with endoscopic removal of the stent. The patient reported resolution of symptoms after removal of the stent.

Revision surgeries range from pyloroplasty, stricturoplasty, and seromyotomy to revision bariatric procedures like gastric bypass [6], single anastomosis gastric bypass, revision sleeve gastrectomy and loop duodenal switch. Dapri et al. [5] and Vilallonga et al. [11] have shown promising results of seromyotomies in patients suffering from long stenosis after sleeve gastrectomies. Some surgeons have approached the refractory strictures with more conservative techniques like a circular gastro-gastrostomy [23] or a laparoscopic median gastrostomy [24]. We had 1 patient who required revision surgeries for complete resolution of symptoms. The patient suffered from a refractory stricture at the incisura 1 month after a LDS procedure. After 3 unsuccessful attempts at graded pneumatic dilation over 6 months, a decision was taken to perform a partial gastrectomy to resect the gastric dilation caused by the stricture. This significantly controlled her symptoms. But one year later, the patient experienced a significant resurgence in her symptoms which led to the decision to perform a Heineke-Mickulicz stricturoplasty with a gastrogastrostomy. This procedure too had limited success. Finally, a decision was taken to place a transabdominal jejunostomy tube. Currently, the patient is still symptomatic but does not want a further intervention and is fine with her limited ability to eat as long as she has a functioning $J$ tube.

In retrospect, our study could have been improved in many ways. These include the addition of a questionnaire to document resolution of symptoms like the BAROS (Bariatric Analysis and Reporting Outcome System) score [25] and a larger sample size.

Another major limitation of our study is the lack of long-term follow up for some of our patients. Though almost all patients at the time of writing do report resolution of symptoms, a follow up of at least 12 months is needed to rule out the possibility of recurrence.

Last but not least due to a small sample size, we were not able to thoroughly explore the management of refractory strictures. Larger studies are needed to compare fcSEMS versus revision surgery for the effective management of refractory strictures. 


\section{Conclusions}

The post-operative stricture is a rare complication following VSG, and earlier detection with effective management significantly reduces patient morbidity. Endoscopic treatment with pneumatic balloon dilation has repeatedly proven to be effective and safe as the first line of management for this complication.

In our series, the duration of dilation is as important as balloon size in achieving early resolution of symptoms and avoiding revision surgeries. However, both the timing and size of the balloon should not be considered settled, and we look for other authors to corroborate our findings or further define what can be done when strictures appear in the sleeve patient. Surgical intervention should be considered only after multiple failed attempts at dilation.

\section{Abbreviations}

BAROS: Bariatric Analysis and Reporting Outcome System; DM: Diabetes mellitus; EBWL: Excess body weight loss; LAGB: Laparoscopic gastric band; LDS: Laparoscopic Duodenal Switch; LSG: Laparoscopic Sleeve Gastrectomy; UGI: Upper gastrointestinal series

\section{Availability of data and materials}

The datasets used and/or analysed during the current study are available from the corresponding author on reasonable request.

\section{Statement of human and animal rights}

All procedures performed in studies involving human participants were in accordance with the ethical standards of the institutional and/or national research committee and with the 1964 Helsinki declaration and its later amendments or comparable ethical standards."

\section{Authors' contributions}

Operating or assistant surgeon and reviewed the manuscript: Drs. DC, CR, and WM. Drafting, data collection, data analysis, and interpretation: AD, AS Data Analysis and interpretation: HZ, AC Manuscript review: AC. All authors read and approved the final manuscript.

\section{Ethics approval and consent to participate}

This study has been approved by Quorum Review- Independent review board (QR\# 31353).

\section{Consent for publication}

Not applicable.

\section{Competing interests}

DC, the corresponding author reports personal fees and other from Medtronic, outside the submitted work.

Drs. $A D, W M, C R, A S$ and $H Z$ declare that they have no competing interests. $A C$ declares that he has no competing interests.

\section{Publisher's Note}

Springer Nature remains neutral with regard to jurisdictional claims in published maps and institutional affiliations.

Received: 7 March 2018 Accepted: 24 July 2018

Published online: 02 August 2018

\section{References}

1. Angrisani L, Santonicola A, lovino P, Formisano G, Buchwald H, Scopinaro N. Bariatric surgery worldwide 2013. Obes Surg. 2015;25(10):1822-32.

2. Zellmer J, Mathiason M, Kallies K, Kothari S. Is laparoscopic sleeve gastrectomy a lower risk bariatric procedure compared with laparoscopic roux-en-Y gastric bypass? A meta-analysis. Am J Surg. 2014;208(6):903-10.

3. Zundel N, Hernandez J, Neto M, Campos J. Strictures after laparoscopic sleeve gastrectomy. Surg Laparosc Endosc Percutan Tech. 2010;20(3):154-8.
4. Eubanks S, Edwards C, Fearing N, Ramaswamy A, de la Torre R, Thaler K, Miedema B, Scott J. Use of endoscopic stents to treat anastomotic complications after bariatric surgery. J Am Coll Surg. 2008;206(5):935-8.

5. Dapri G, Cadière G, Himpens J. Laparoscopic Seromyotomy for long stenosis after sleeve gastrectomy with or without duodenal switch. Obes Surg. 2009; 19(4):495-9.

6. Bellorin O, Lieb J, Szomstein S, Rosenthal R. Laparoscopic conversion of sleeve gastrectomy to roux-en-Y gastric bypass for acute gastric outlet obstruction after laparoscopic sleeve gastrectomy for morbid obesity. Surg Obes Relat Dis. 2010;6(5):566-8.

7. Cottam A, Cottam D, Roslin M, Cottam S, Medlin W, Richards C, Surve A, Zaveri $\mathrm{H}$. A matched cohort analysis of sleeve gastrectomy with and without $300 \mathrm{~cm}$ loop duodenal switch with 18-month follow-up. Obes Surg. 2016;26(10):2363-9.

8. Rosenthal R. International sleeve gastrectomy expert panel consensus statement: best practice guidelines based on experience of $>12,000$ cases. Surg Obes Relat Dis. 2012:8(1):8-19.

9. Cottam D, Qureshi F, Mattar S, Sharma S, Holover S, Bonanomi G, Ramanathan R, Schauer P. Laparoscopic sleeve gastrectomy as an initial weight-loss procedure for high-risk patients with morbid obesity. Surg Endosc. 2006;20(6):859-63.

10. Shnell M, Fishman S, Eldar S, Goitein D, Santo E. Balloon dilatation for symptomatic gastric sleeve stricture. Gastrointest Endosc. 2014;79(3):521-4.

11. Vilallonga R, Himpens J, van de Vrande S. Laparoscopic Management of Persistent Strictures after Laparoscopic Sleeve Gastrectomy. Obes Surg. 2013:23(10):1655-61.

12. Donatelli G, Dumont J, Pourcher G, Tranchart H, Tuszynski T, Dagher I, Catheline J, Chiche R, Marmuse J, Dritsas S, Vergeau B, Meduri B. Pneumatic dilation for functional helix stenosis after sleeve gastrectomy: long-term follow-up (with videos). Surg Obes Relat Dis. 2016;13(6):943-50.

13. Rebibo L, Hakim S, Dhahri A, Yzet T, Delcenserie R, Regimbeau J. Gastric stenosis after laparoscopic sleeve gastrectomy: diagnosis and management. Obes Surg. 2015;26(5):995-1001.

14. Carlin AM, Arthur M, et al. The Comparative Effectiveness Of Sleeve Gastrectomy, Gastric Bypass, And Adjustable Gastric Banding Procedures For The Treatment Of Morbid Obesity. Annals of Surgery. 2013;257(5):791-7.

15. Casillas RA, et al. Comparative Effectiveness Of Sleeve Gastrectomy Versus Roux-En-Y Gastric Bypass For Weight Loss And Safety Outcomes In Older Adults. Surgery for Obesity and Related Diseases. 2017;13(9):1476-83.

16. Boeckxstaens G, Annese V, Varannes S, Chaussade S, Costantini M, Cuttitta A Elizalde J, Fumagalli U, Gaudric M, Rohof W, Smout A, Tack J, Zwinderman A, Zaninotto G, Busch O. Pneumatic dilation versus laparoscopic Heller's Myotomy for idiopathic achalasia. N Engl J Med. 2011;364(19):1807-16.

17. Gambardella C, Allaria A, Siciliano G, et al. Recurrent esophageal stricture from previous caustic ingestion treated with 40-year self-dilation: case report and review of literature. BMC gastroenterol. 2018;18(1):68.

18. Ogra R, Kini G. Evolving endoscopic management options for symptomatic stenosis post-laparoscopic sleeve gastrectomy for morbid obesity: experience at a large bariatric surgery unit in New Zealand. Obes Surg. 2014:25(2):242-8.

19. Deslauriers V, Beauchamp A, Garofalo F, et al. Endoscopic management of post-laparoscopic sleeve gastrectomy stenosis. Surg Endosc. 2018; 32(2):601-9.

20. Nath A, Yewale S, Tran T, Brebbia JS, Shope TR, Koch TR. Dysphagia after vertical sleeve gastrectomy: evaluation of risk factors and assessment of endoscopic intervention. World J Gastroenterol. 2016;22(47):10371-9.

21. Agnihotri A, Barola S, Hill C, et al. Endoscopic management of gastric stenosis following laparoscopic sleeve gastrectomy. GIE. 2017;(85, 5):AB276.

22. Sudan R, Kasotakis G, Betof A, Wright A. Sleeve gastrectomy strictures: technique for robotic-assisted strictureplasty. Surg Obes Relat Dis. 2010;6(4): 434-6.

23. Parikh M, Gagner M. Laparoscopic revision of Gastrogastric stricture with a Transoral circular stapler. Surg Innov. 2007;14(3):225-30.

24. Kalaiselvan $\mathrm{R}$, Ammori B. Laparoscopic median gastrectomy for stenosis following sleeve gastrectomy. Surg Obes Relat Dis. 2015;11(2):474-7.

25. Oria $\mathrm{H}$, Moorehead M. Updated bariatric analysis and reporting outcome system (BAROS). Surg Obes Relat Dis. 2009;5(1):60-6. 